\title{
Leishmania enriettii: biochemical characterisation of lipophosphoglycans (LPGs) and glycoinositolphospholipids (GIPLs) and infectivity to Cavia porcellus
}

Larissa Ferreira Paranaíba ${ }^{1,2}$, Rafael Ramiro de Assis ${ }^{1}$, Paula Monalisa Nogueira ${ }^{1,5}$, Ana Claúdia Torrecilhas ${ }^{4}$, João Henrique Campos ${ }^{4}$, Amanda Cardoso de Oliveira Silveira', Olindo Assis Martins-Filho', Natalia Lima Pessoa”, Marco Antônio Campos ${ }^{1}$, Patrícia Martins Parreiras ${ }^{1}$, Maria Norma Melo ${ }^{3}$, Nelder de Figueiredo Gontijo² and Rodrigo Pedro Pinto Soares ${ }^{1,5^{*}}$

\begin{abstract}
Background: Leishmania enriettii is a species non-infectious to man, whose reservoir is the guinea pig Cavia porcellus. Many aspects of the parasite-host interaction in this model are unknown, especially those involving parasite surface molecules. While lipophosphoglycans (LPGS) and glycoinositolphospholipids (GIPLs) of Leishmania species from the Old and New World have already been described, glycoconjugates of $L$. enriettii and their importance are still unknown.

Methods: Mice peritoneal macrophages from C57BL/6 and knock-out (TLR2 -/-, TLR4 -/-) were primed with IFN- $\gamma$ and stimulated with purified LPG and GIPLs from both species. Nitric oxide and cytokine production were performed. MAPKs (p38 and JNK) and NF-kB activation were evaluated in J774.1 macrophages and CHO cells, respectively.

Results: LPGs were extracted, purified and analysed by western-blot, showing that LPG from L88 strain was longer than that of Cobaia strain. LPGs and GIPLs were depolymerised and their sugar content was determined. LPGs from both strains did not present side chains, having the common disaccharide Gal( $(31,4) M a n(a 1)-P O_{4}$. The GIPL from L88 strain presented galactose in its structure, suggestive of type II GIPL. On the other hand, the GIPL of Cobaia strain presented an abundance of glucose, a characteristic not previously observed. Mice peritoneal macrophages from C57BL/6 and knock-outs (TLR2 -/- and TLR4 -/-) were primed with IFN- $\gamma$ and stimulated with glycoconjugates and live parasites. No activation of NO or cytokines was observed with live parasites. On the other hand, LPGs and GIPLs were able to activate the production of NO, IL-6, IL-12 and TNF-a preferably via TRL2. However, in CHO cells, only GIPLs were able to activate TRL2 and TRL4. In vivo studies using male guinea pigs (Cavia porcellus) showed that only strain L88 was able to develop more severe ulcerated lesions especially in the presence of salivary gland extract (SGE).
\end{abstract}

Conclusion: The two L. enriettii strains exhibited polymorphisms in their LPGs and GIPLs and those features may be related to a more pro-inflammatory profile in the L88 strain.

Keywords: Leishmania enriettii, Glycoconjugates, Lipophosphoglycan, Glycoinositolphospholipids, Cavia porcellus, Macrophage, Innate immunity

\footnotetext{
*Correspondence: rsoares@cpqrr.fiocruz.br

${ }^{1}$ Centro de Pesquisas René Rachou, Fundação Oswaldo Cruz, FIOCRUZ, Belo Horizonte, Minas Gerais, Brazil

${ }^{5}$ Laboratory of Cellular and Molecular Parasitology, Centro de Pesquisas René Rachou, Fundação Oswaldo Cruz, FIOCRUZ, Av. Augusto de Lima 1715, Belo Horizonte, Minas Gerais 30190-002, Brazil

Full list of author information is available at the end of the article
} 


\section{Background}

The leishmaniases are diseases caused by the protozoa Leishmania (Kinetoplastida, Trypanosomatidae) transmitted to the vertebrate host by phlebotomine sand flies (Psychodidae: Phlebotominae). There are over 30 species of Leishmania in the New World, where Leishmania enriettii Muniz and Medina 1948 is an example of a non-infectious species to man [1]. Its vertebrate host is the guinea pig Cavia porcellus, and its suspected vector is Lutzomyia monticola [2,3].

Although non-infectious for humans, Leishmania enriettii is considered a model for the human cutaneous leishmaniasis (CL) [4-6]. An interesting aspect of this species is that it had never been found in any continent other than the Americas. However, kinetoplastid parasites from the Leishmania enriettii complex were recently found in infected Red Kangaroo (Macropus rufus) in Australia. In this context, the vector species was Forcipomyia lasiohelea, a ceratopogonid [7].

Glycoconjugates are very important during the interaction with the vertebrate and invertebrate hosts. In the New World species, the early events in the innate immune compartment are crucial for the development of a response against the parasite [8]. Glycoconjugates can be associated with glycosylphosphatidylinositol (GPI) anchors in the plasma membrane, especially the lipophosphoglycans (LPGs), gp63 and glycolinositolphospholipids (GIPLs) [9]. GIPLs are present on the cell surface in all stages and represent the most abundant glycoconjugates [9]. Structurally, GIPLs are widely polymorphic, but with a basic conserved structure of Mano1-4GlcN linked to the lipid portion, which usually consists of alkylacylglycerol or lysoalkylglycerol (lyso-PI), by means of a phosphatidylinositol residue (PI) [10] and can be classified into three groups: Type I GIPLs have, for the greater part, a mannose residue as the most distal sugar, and can be identified by a substitution of the sixth carbon of the proximal mannose by a mannose residue (Man 1 1-6Man $\alpha 1-4 G l c N-P I)$. Type II GIPLs are characterised by the substitution of the third carbon of the proximal mannose by a mannose residue (Mano1-3Man 1 1-4GlcN-PI). The third group (hybrid GIPLs), shares structural features with the first two, possessing one additional mannose in the third and sixth

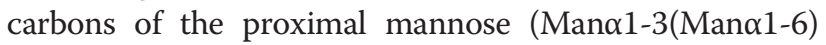
Mano1-4GlcN-PI). Recently, Leishmania infantum and Leishmania braziliensis GIPLs were preliminarily characterised, as being of type I and Type II, respectively. An interesting feature of those molecules is their inhibitory properties via TLR4 during the interaction with $\mathrm{BALB} / \mathrm{c}$ and C57BL/6 macrophages [11].

LPG is the most studied glycoconjugate in Leishmania. It is widely expressed in the surface of promastigote forms having four domains: a lipid anchor (1-O-alkyl-2lysophosphatidylinositol); a heptasaccharide core (Gal( $\alpha 1-6)$
$\mathrm{Gal}(\alpha 1-3) \mathrm{Gal}_{\mathrm{f}}(\alpha 1-3)\left[\mathrm{Glc} \alpha 1-\mathrm{PO}_{4}\right] \operatorname{Man}(\alpha 1-3) \operatorname{Man}(\alpha 1-4)-$ $\operatorname{GlcN}(\alpha 1-))$; a region of repeat units $(\mathrm{Gal}(\beta 1-4) \mathrm{Man}$ $\left.(\alpha 1-) \mathrm{PO}_{4}\right)$ and the terminal neutral oligosaccharide cap [12]. LPG has been involved in a wide variety of functions including recognition, phagocytosis and protection from the acidic environment of parasitophorous vacuoles $[13,14]$, resistance to complement, inhibition of phagosome maturation [15], inhibition of protein kinase C [16], induction of protein kinease $\mathrm{R}$ [17], the ability to intervene in the integrity of microdomains in phagosomal plasma membranes [18], modulation of nitric oxide (NO) and IL-12 production [16,19-21], modulation of MAPKs [21,22], agonist of TLR2 and TLR4 [17,21,23-25], induction of neutrophil extracellular traps (NETs) [26] and heme-oxigenase 1 [27] and attachment to the sand fly midgut [28-31].

Many studies have explored the role of Leishmania during the interaction with the vertebrate host [32]. However, most of those studies focused on Old World species of Leishmania. Recently, it was demonstrated that the LPG of $L$. braziliensis was more pro-inflammatory than that of L. infantum, suggesting that polymorphisms in the LPG structures may be important during the immunopathology of the disease. In C57BL/6 macrophages (and respective knock-outs) and CHO cells a predominant role of TLR2 was shown [21]. However, those and many other aspects are still unknown in L. enriettii.

In this work, we have studied two reference strains of L. enriettii isolated from C. porcellus in two distinct moments (1945 and 1985). This study aimed to preliminary characterise their glycoconjugates (LPG and GIPLs) and their role during in vitro interaction with macrophages and $\mathrm{CHO}$ cells. Additionally, their infectivity was tested with its natural vertebrate host in the presence and absence of salivary gland extract (SGE) from Lutzomyia longipalpis. This is part of a wider study on the glycobiology of New World species of Leishmania.

\section{Methods}

\section{Ethics statement}

All animals were handled in strict accordance with animal practice as defined by the Internal Ethics Committee in Animal Experimentation (CEUA) of Fundação Oswaldo Cruz (FIOCRUZ), Belo Horizonte (BH), Minas Gerais (MG), Brazil (Protocol p-0297-06). Knock-out mice handling protocol was approved by the National Commission of Biosafety (CTNBio) (protocol \#01200.006193/2001-16).

\section{Parasites}

World Health Organization Reference strains of $L$. enriettii (MCAV/BR/1945/L88 and MCAV/BR/1985/COBAIA_SP) were used. Promastigotes were cultured in M199 medium supplemented with $10 \%$ heat inactivated fetal bovine serum (FBS), penicillin 100 units $/ \mathrm{mL}$, streptomycin $50 \mu \mathrm{g} / \mathrm{mL}$, 
$12.5 \mathrm{mM}$ glutamine, $0.1 \mathrm{M}$ adenine, $0.0005 \%$ hemin, and $40 \mathrm{mM}$ Hepes, $\mathrm{pH} 7.4$ at $26^{\circ} \mathrm{C}$ until late log phase [33].

\section{Extraction and purification of LPGs and GIPLs}

For optimal LPGs and GIPLs extractions, late log phase cells were harvested and washed twice with PBS prior to extraction of LPGs and GIPLs. The LPGs and GIPLs extractions were performed as described elsewhere with solvent $\mathrm{E} \quad\left(\mathrm{H}_{2} \mathrm{O} /\right.$ ethanol/diethylether/pyridine $/ \mathrm{NH}_{4} \mathrm{OH}$; 15:15:5:1:0.017) after a sequential organic solvent extraction [12]. For purification, the solvent $\mathrm{E}$ extract was dried under $\mathrm{N}_{2}$ evaporation, resuspended in $2 \mathrm{~mL}$ of $0.1 \mathrm{~N}$ acetic acid/0.1 M NaCl, and applied onto a column with $2 \mathrm{~mL}$ of phenyl-Sepharose, equilibrated in the same buffer. The column was washed with $6 \mathrm{ml}$ of $0.1 \mathrm{~N}$ acetic acid/ $0.1 \mathrm{M} \mathrm{NaCl}$, then $1 \mathrm{~mL}$ of $0.1 \mathrm{~N}$ acetic acid and finally $1 \mathrm{~mL}$ of endotoxin free water. The LPGs and GIPLs were eluted with $4 \mathrm{~mL}$ of solvent $\mathrm{E}$, dried under $\mathrm{N}_{2}$ evaporation and quantitated as described [34]. Prior to use on in vitro macrophage cultures, LPG and GIPLs were diluted in fresh RPMI. All solutions were prepared in sterile, LPS-free distilled water (Sanobiol, Campinas, Brazil). All experimental procedures are depicted in (Figure 1).

\section{Fluorophore-assisted carbohydrate electrophoresis (FACE) Oligosaccharides}

Phosphorylated repeat units were treated with alkaline phosphatase in $15 \mathrm{mM}$ Tris buffer, pH 9.0 (1 U, $16 \mathrm{~h}$, $37^{\circ} \mathrm{C}$ ) (Figure 1). Samples were desalted and subjected to fluorophore-assisted carbohydrate electrophoresis (FACE). Samples were fluorescently labelled with 0.05 N ANTS (8-aminonaphthalene-1,3,6-trisulfate) and $1 \mathrm{M}$ cyanoborohydride $\left(37^{\circ} \mathrm{C}, 16 \mathrm{~h}\right)$. Oligoglucose ladders $\left(\mathrm{G}_{1}-\mathrm{G}_{7}\right)$ were used as standards. Sugars were subjected to FACE and the gel was visualised by an UV imager [35].

\section{Monosaccharides}

To access the monosaccharide composition, deaminated GIPLs headgroups were subjected to strong acid hydrolysis $\left(2 \mathrm{~N}\right.$ trifluoroacetic acid, $\left.3 \mathrm{~h}, 100^{\circ} \mathrm{C}\right)$ (Figure 1$)$. To determine the monosaccharide composition of the GIPLs, depolymerised and desalted monosaccharides were fluorescently labeled with 0.1 M AMAC (2-aminoacridone) in $5 \%$ acetic acid and $1 \mathrm{M}$ cyanoborohydride. Labeled sugars were subjected to FACE and the gel was visualized under UV light. Monosaccharides (D-galactose, D-glucose and D-mannose) (Sigma) were used as standards [11].

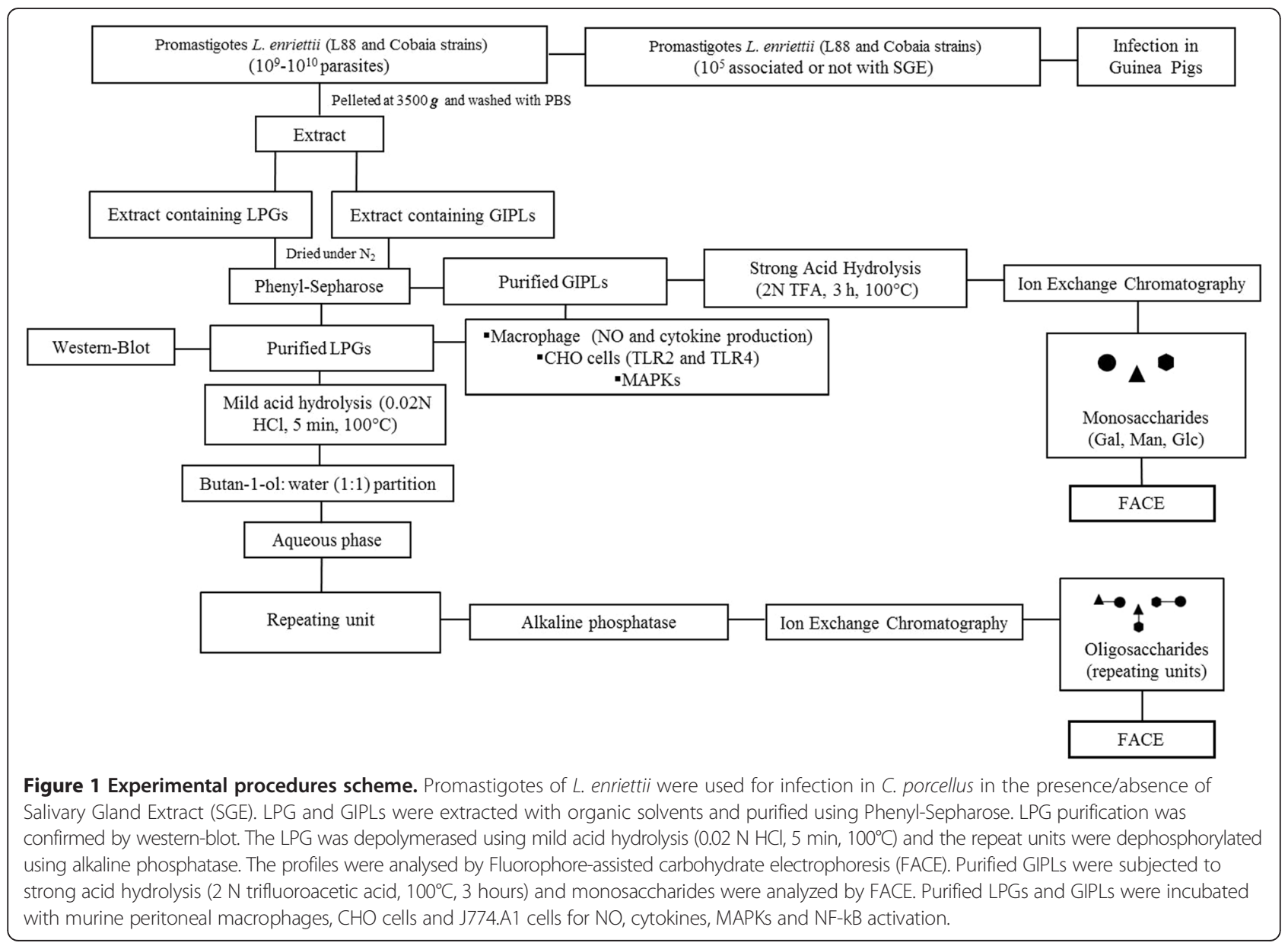




\section{Purification of murine peritoneal macrophages and cell culture}

Thioglycollate-elicited peritoneal macrophages were extracted from C57BL/6 and C57BL/6 (TLR2-/- and TLR4-/- knockouts) by peritoneal washing with ice cold serum-free RPMI and enriched by plastic adherence for $1 \mathrm{~h}$ at $37^{\circ} \mathrm{C} / 5 \% \mathrm{CO}_{2}$. Cells $\left(3 \times 10^{5}\right.$ cells/well $)$ were washed with fresh RPMI and cultured in RPMI, 2 mM glutamine, $50 \mathrm{U} / \mathrm{mL}$ of penicillin and $50 \mu \mathrm{g} / \mathrm{mL}$ streptomycin supplemented with $10 \%$ FBS in 96-well culture plates $\left(37^{\circ} \mathrm{C} / 5 \% \mathrm{CO}_{2}\right)$. Cells were primed with gamma interferon $(\mathrm{IFN}-\gamma)(3 \mathrm{IU} / \mathrm{mL})$ [36] for $18 \mathrm{~h}$ prior to incubation with $L$. enriettii LPGs $(10 \mu \mathrm{g} / \mathrm{mL})$, GIPLs $(10 \mu \mathrm{g} / \mathrm{mL})$, L. braziliensis LPG $(10 \mu \mathrm{g} / \mathrm{mL})$, lipopolysaccharide (LPS) $(100 \mathrm{ng} / \mathrm{mL})$ or live stationary L. enriettii parasites (MOI 10:1).

\section{Cytokine and nitrite measurements}

For CBA multiplex cytokine detection, cells were plated, primed as described above and incubated with LPGs, GIPLs, LPS and live stationary promastigotes (MOI 10:1) for 48 h. Leishmania braziliensis LPG $(10 \mu \mathrm{g} / \mathrm{mL})$ was added as a positive control [21]. For negative controls fresh medium was added. Supernatants were collected and IL1- $\beta$, IL-6, IL-10, IL-12p40 and TNF- $\alpha$ were determined using the BD CBA Mouse Cytokine assay kits according to the manufacturer's specifications (BD Biosciences, CA, USA). Flow cytometry measurements were performed on a FACS Calibur flow cytometer (Becton Dickinson, Mountain View, CA). Cell-Quest ${ }^{\text {mM }}$ software package provided by the manufacturer was used for data acquisition and the FlowJo software 7.6.4 (Tree Star Inc., Ashland, OR, USA) was used for data analysis. A total of 2,400 events were acquired for each preparation. Results are representative of two experiments in duplicate. Nitrite concentrations were determined by Griess reaction [21].

\section{Chinese Hamster Ovary (CHO) cell lines}

The CHO reporter cell lines TLR2-TLR4-, which do not express TLR2 nor TLR4; TLR2+, expressing TLR2 and TLR4+, expressing TLR4 [37] were maintained as adherent monolayers in Ham's F-12/DMEM supplemented with $5 \% \mathrm{FBS}$, at $37^{\circ} \mathrm{C} / 5 \% \mathrm{CO}_{2}$, and antibiotics. All cell lines were derived from clone $3 \mathrm{E} 10$, that has been stably transfected with a reporter construct containing the structural gene for CD25 under the control of the human E-selectin promoter. This promoter contains a NF$\mathrm{kB}$ binding site; CD25 surface expression is completely dependent upon NF-kB translocation to the cell nucleus $[21,38]$. In order to evaluate the activation of NF-kB by LPGs and GIPLs, CHO reporter cells were plated at a density of $1 \times 10^{5}$ cells/well in 24-well tissue culture dishes. The following day, either molecule or bacteria
(Staphylococcus aureus $\left[10^{3}\right.$ bacteria/well], positive control of TLR2; LPS (200 ng/well), positive control of TLR4; or LPGs $(0.2 \mu \mathrm{g}$ or $0.02 \mu \mathrm{g} /$ well $)$ and GIPLs $(0.2 \mu \mathrm{g}$ or $0.02 \mu \mathrm{g} /$ well $)$ from the two L. enriettii strains were added for $18 \mathrm{~h}$. LPGs from L. braziliensis and $L$. infantum were used as positive and negative controls, respectively [21]. The cells were harvested with trypsinEDTA, washed with medium and PBS. Subsequently, $1 \times 10^{5}$ were cells were stained with PE-labeled antiCD25 (mouse mAb to human CD25, R-PE conjugate; Caltag Laboratories, Burlingame, CA) 1:200 in PBS, on ice, in the dark, for $30 \mathrm{~min}$. After labeling, the cells were washed twice with the same buffer, resuspended in $1 \mathrm{mM}$ sodium azide in PBS, and examined by flow cytometry (BD Biosciences, San Jose, CA) for the expression of surface CD25 as described [37]. Analyses were performed using Cell Quest software (BD Biosciences).

\section{Activation of MAPKs}

We investigated whether LPGs and GIPLs from the two L. enriettii strains could modulate MAPKs activation. J774.1 macrophages were plated as above on 24 well tissue culture plates $\left(3 \times 10^{6} /\right.$ well $)$ for $18 \mathrm{~h}$ prior to assay [21]. The cells were washed with warm RPMI and incubated with LPG and GIPLs from both strains for different times $(5,15,30$ and $45 \mathrm{~min})$ or with medium (negative control) or LPS $(100 \mathrm{ng} / \mathrm{ml})$ as positive control. Cells were then washed with ice-cold PBS and lysed in RIPA lysis buffer (Sigma) and protease inhibitor cocktail (Thermo Scientific). Cells were harvested with a plastic scraper and centrifuged at $13,000 \times \mathrm{g}\left(4^{\circ} \mathrm{C}, 10 \mathrm{~min}\right)$. Supernatants were transferred to new tubes and stored at $-20^{\circ} \mathrm{C}$ until used for immunoblotting. Cell lysates were resolved by SDS-PAGE, transferred to a nitrocellulose membrane and blocked (5\% milk in TBS-0.1\% Tween 20) for $1 \mathrm{~h}$. Primary Abs [dually phosphorylated p38 (Santa Cruz) and JNK (Sigma) 1:1,000, total p38 primary antibody (Sigma) was used as a normalizer] were incubated for $16 \mathrm{~h}$ at $4^{\circ} \mathrm{C}$. Membranes were washed $(3 \times 10 \mathrm{~min})$ with TBS-0.1\% Tween 20 and incubated $1 \mathrm{~h}$ with anti-mouse IgG conjugated with peroxidase $(1: 10,000)$. The reaction was visualised using luminol [21]. The data were analysed by Densitometry using the software Image $1.48 \mathrm{v}$ by National Institutes of Health (http://imagej.nih.gov/ij).

\section{Sand flies}

Lutzomyia longipalpis sand flies were captured in Teresina, Piauí state, Brazil. The insects were reared in the Laboratório de Fisiologia de Insetos Hematófagos at the Universidade Federal de Minas Gerais in Brazil using existing methodology [39]. Three to 6-day-old non-fed female sand flies, maintained on $30 \%$ sucrose were dissected in PBS. Heads, crops, hindguts, and Malpighian tubules were removed, and the isolated salivary glands were dissected. Ten salivary 
glands were dissected, placed in $20 \mu \mathrm{L}$ of PBS and stored at $-80^{\circ} \mathrm{C}$. Immediately before use, the glands were sonicated for 10 seconds in a water bath [40] and centrifuged $(2100 \mathrm{~g}, 10 \mathrm{~min})$. The supernatant containing Salivary Gland Extract (SGE) was mixed with $1 \times 10^{6}$ parasites $/ \mathrm{ml}$ in PBS prior to in vivo experiments.

\section{In vivo infections with guinea pigs}

For intradermal inoculation, we used $1 \times 10^{5}$ L. enriettii promastigotes in a volume of $100 \mu \mathrm{l}$ in PBS [40], associated or not with SGE. Forty males of Cavia porcellus (20 per experiment) were divided into 4 groups: group 1 (infected with L88 strain), group 2 (infected with L88 strain + SGE of L. longipalpis), group 3 (infected with Cobaia strain) and group 4 (infected with Cobaia strain + SGE of L. longipalpis). The course of infection was assessed weekly for 91 days by measuring the lesion areas $\left(\mathrm{mm}^{2}\right)$.

\section{Statistical analyses}

For nitrite and cytokine measurements, the Shapiro Wilk test was conducted to test the null hypothesis that data were sampled from a Gaussian distribution (SHAPIRO, 1965). The $P$ value $(P>0.05)$ showed that data did not deviate from Gaussian distribution. For this reason, Student's " $t$ " test and ANOVA were performed to test equality of population medians among groups and independent samples. Data were analyzed using GraphPad Prism 5.0 software (Graph Prism Inc., San Diego, CA) and $\mathrm{P}<0.05$ was considered significant.

\section{Results}

\section{Growth curve}

A growth curve was established to determine the division profile of the parasites for glycoconjugate extraction. The strains showed similar growth patterns reaching the stationary phase after the $10^{\text {th }}$ day. Cobaia reached higher densities than L88 $\left(8.7 \times 10^{6}\right.$ versus $6.5 \times 10^{6}$ parasites $\left./ \mathrm{mL}\right)$ $(\mathrm{P}<0.05)$. For this reason, we selected the $10^{\text {th }}$ day of growth for LPG and GIPLs extraction for both strains.

\section{LPG purification}

In Western Blots, purified LPGs from both L. enriettii strains were recognized by the antibody CA7AE, whose structural epitope is the unbranched $\mathrm{Gal}(\beta 1,4) \mathrm{Man}\left(\alpha 1-\mathrm{PO}_{4}\right)$ repeat units, common to all LPGs [41]. The LPG of the L88 strain showed a higher molecular weight smear (upper arrow) in Western Blots than that of the Cobaia strain (lower arrow), suggesting that the former possessed a longer LPG. As expected, the positive control (LPG of the BH46 L. infantum strain) [42] was recognised by the antibody (Figure 2).

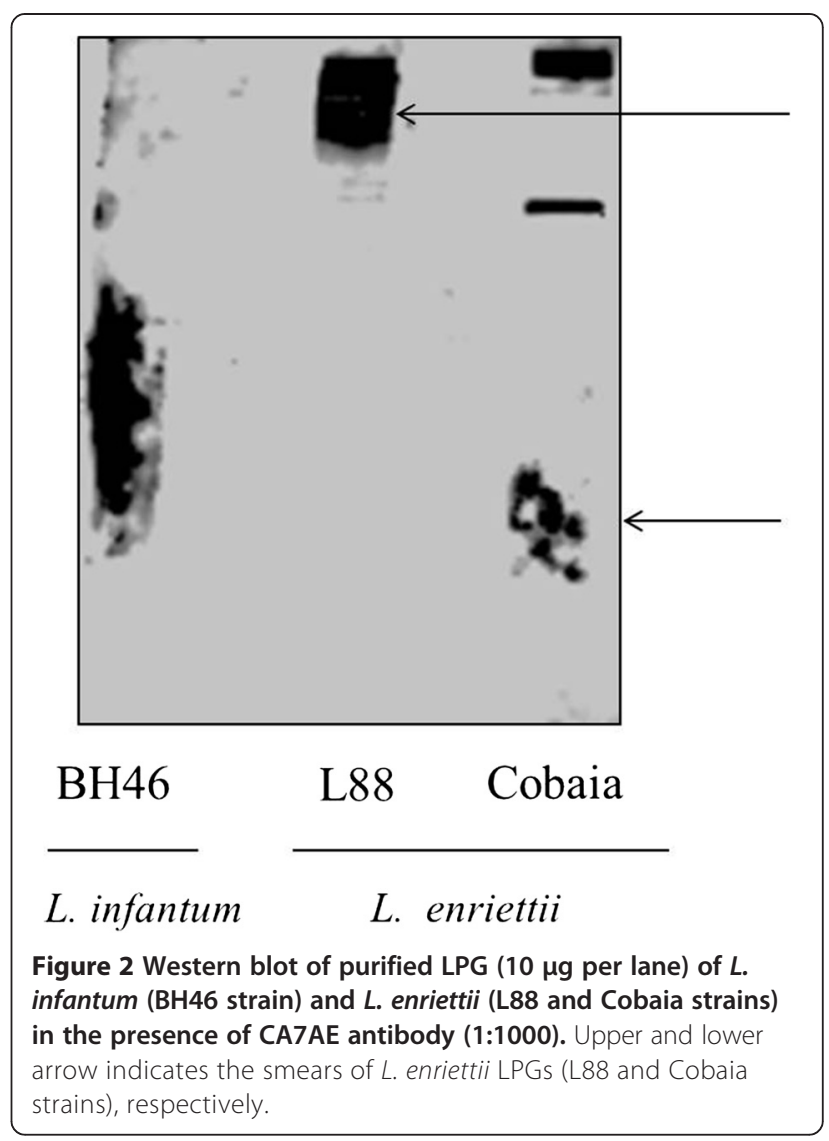

\section{Profile analysis of the LPG repeat units}

All LPGs were subjected to mild acid hydrolysis to obtain their repeat units (Figure 1). These units were tagged with a fluorescent probe at the reducing ends and subjected to FACE for visualisation of the carbohydrate profiles. In order to identify the number of sugars, we used a standard molecular weight of oligoglucoses $\left(G_{1}-G_{7}\right)$, whose bands indicate the migration profile from mono to heptasaccharides (Figure 3A, STD lane). Both strains showed a band at the $G_{2}$ position, an expected result since all LPGs possess the Gal-Man disaccharide (Figure 3A). Therefore, these data indicate that the LPGs from both $L$. enriettii strains are devoid of side chains.

\section{Identification of GIPL monosaccharides}

In order to determine the monosaccharide composition of GIPLs, the purified GIPLs were subjected to strong acid hydrolysis (Figure 1B). Unlike what was observed in the polysaccharide gel (Figure $3 \mathrm{~A}$ ), there was a polymorphism in the GIPLs composition. As expected, mannose and galactose are present in both strains of $L$. enriettii. The L88 strain is characterised by being rich in the monosaccharide galactose, suggesting similarity with the type II GIPL [11]. On the other hand, the structure of the GIPL from the Cobaia strain contains the sugars mannose and glucose in its composition. It is noteworthy that this GIPL contains 


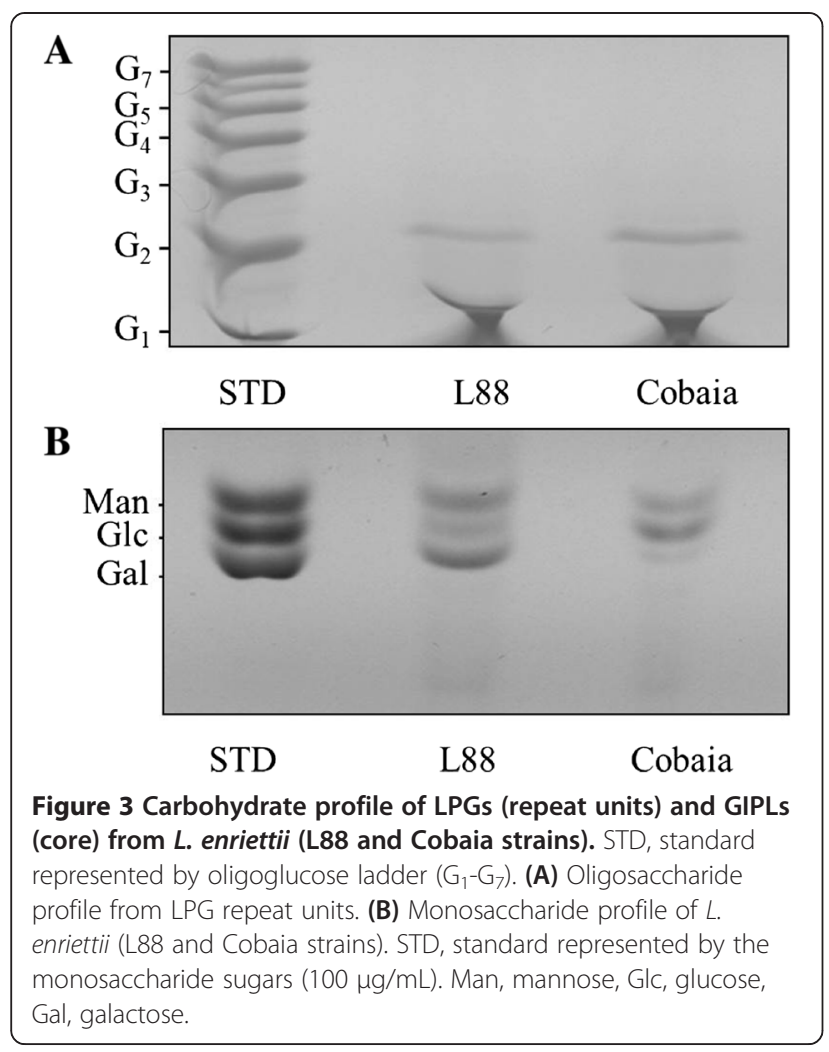

glucose, a feature never observed in other Leishmania GIPLs (Figure 3B).

\section{Analysis of NO production in murine macrophages}

In order to determine the NO production profile in peritoneal macrophages of the C57BL/6, TLR2 (-/-) and TLR4 (-/-) lineages, and possible participation of TLRs, these cells were stimulated with LPGs, GIPLs, LPS and promastigote forms from both $L$. enriettii strains. No activation was observed for the living parasites, growth medium or IFN- $\gamma$ (Figure 4A). There was no statistical difference regarding NO synthesis in the wild mice stimulated with LPGs from both strains. However, a higher NO production was observed in macrophages incubated with LPGs (Knock-outs) and GIPLs (all lineages) from L88 strain $(\mathrm{P}<0.05)$. This activation was primarily via TLR2, and secondarily via TLR4 $(\mathrm{P}<0.0001)$ (Figure $4 \mathrm{~A})$.

\section{Analysis of cytokine production in murine macrophages}

Similarly to the NO experiment, supernatants from macrophage cultures of the three strains of mice (C57BL/6, TLR2 (-/-) and TLR4 (-/-)) were subjected to CBA analysis to quantitate the cytokines TNF- $\alpha$, IL- $1 \beta$, IL-6, IL-10 and IL-12p40. Similar to previous results with other Leishmania species [11,21], no cytokine production by living parasites from both strains was observed (Figure 4B; Figure 5). A higher pro-inflammatory activity was observed for the LPG and GIPL from L88 strain, where higher amounts $(\mathrm{pg} / \mathrm{ml})$ of the citokines TNF- $\alpha$, IL6 and IL-12p40 were detected $(\mathrm{P}<0.05)$. Similarly to NO, this production was primarily via TLR2 than TLR4, especially for TNF- $\alpha$ (Figure 4B). No significant levels were detected for the cytokines IL-10 and IL-1 $\beta$ in any of the experiments (data not shown).

\section{Analysis of NF-kB translocation by LPGs and GIPLs}

Considering that TLR2 and TLR4 were the main receptors recognising the LPGs and GIPLs from both L. enriettii strains, the role of each of these receptors was assessed separately in $\mathrm{CHO}$ cells. These cells were treated with LPGs and GIPLs for 18 hours and the expression of the CD25 reporter was analysed by flow cytometry [21]. No activation of TLR2 and TLR4 receptors was observed after incubation with LPG $(0.2$ and $0.02 \mu \mathrm{g})$. However, a higher activation of GIPLs, especially in TLR2 than TLR4 was observed (Figure 6). As expected, the positive control groups for TLR2, represented by the LPG of L. braziliensis and the lysate of $S$. aureus, activated the translocation of NF-kB [21]. The positive control group for TLR4, represented by LPS, also activated NF-kB. Negative control represented by LPG of L. infantum (BH46 strain) [21], did not activate NF-kB (Figure 6).

\section{Activation of MAPKs}

To better access the signaling events around LPGs and GIPLs recognition and macrophage activation, J774.1 macrophages were incubated with L. enriettii (L88 and Cobaia strains) LPGs and GIPLs. MAPK activation was assessed as a function of time and analysed by Densitometry. Similarly to cytokine production, the LPG of L88 strain was able to activate JNK more pronounced than Cobaia strain (Figure 7A and B). No differences in p38 activation were observed for LPG and GIPLs between the two strains (Figure 7C and D).

\section{Infection in Cavia porcellus using the two L. enriettii strains}

Since glycoconjugates (LPG and GIPLs) from the two $L$. enriettii strains exhibited a distinct pro-inflammatory profile during in vitro experiments, live parasites were inoculated in C. porcellus for evaluation of their infectivity.

No mortality was observed during the experiment (91 days). There was no statistical difference between the weights of groups 1 and 2, and between groups 3 and 4 throughout the experiment $(P>0.05)$. In spite of the absence of lesions, all Cobaia infected animals exhibited protuberances (Figure $8 \mathrm{~F}$ ). For this reason, lesion analysis was restricted to L88 strain (Figures 8A-E and Figure 9). Infected animals started developing protuberances and/or lesions during the fourth-fifth week of infection (Figure 9). The presence of SGE resulted in an increase of lesion size $\left(477 \pm 220.2\right.$ versus $\left.294.2 \pm 120.0 \mathrm{~mm}^{2}\right)$ 


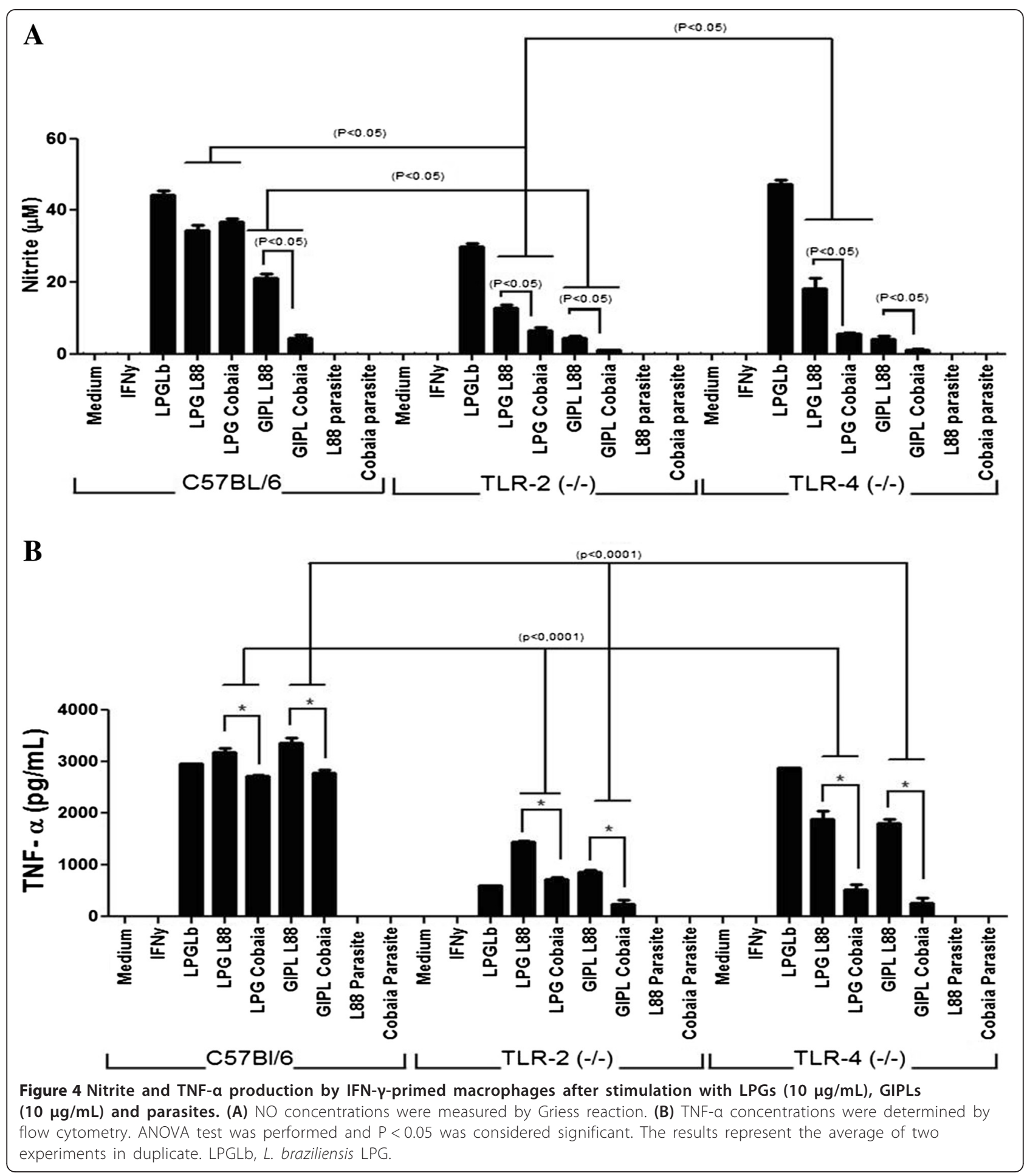

$(\mathrm{P}<0.05)$. Also, a greater onset-healing period (ten weeks) was observed for SGE injected animals. After this period, a gradual decrease in the area of the lesion was noticed with a complete absence of lesion after the thirteenth week (Figure 9).

\section{Discussion}

This work compared two L. enriettii strains isolated from the wild between a 40-year interval. The parameters studied included: growth curve, biochemical analysis of glycoconjugates (LPGs and GIPLs), NO and cytokine 


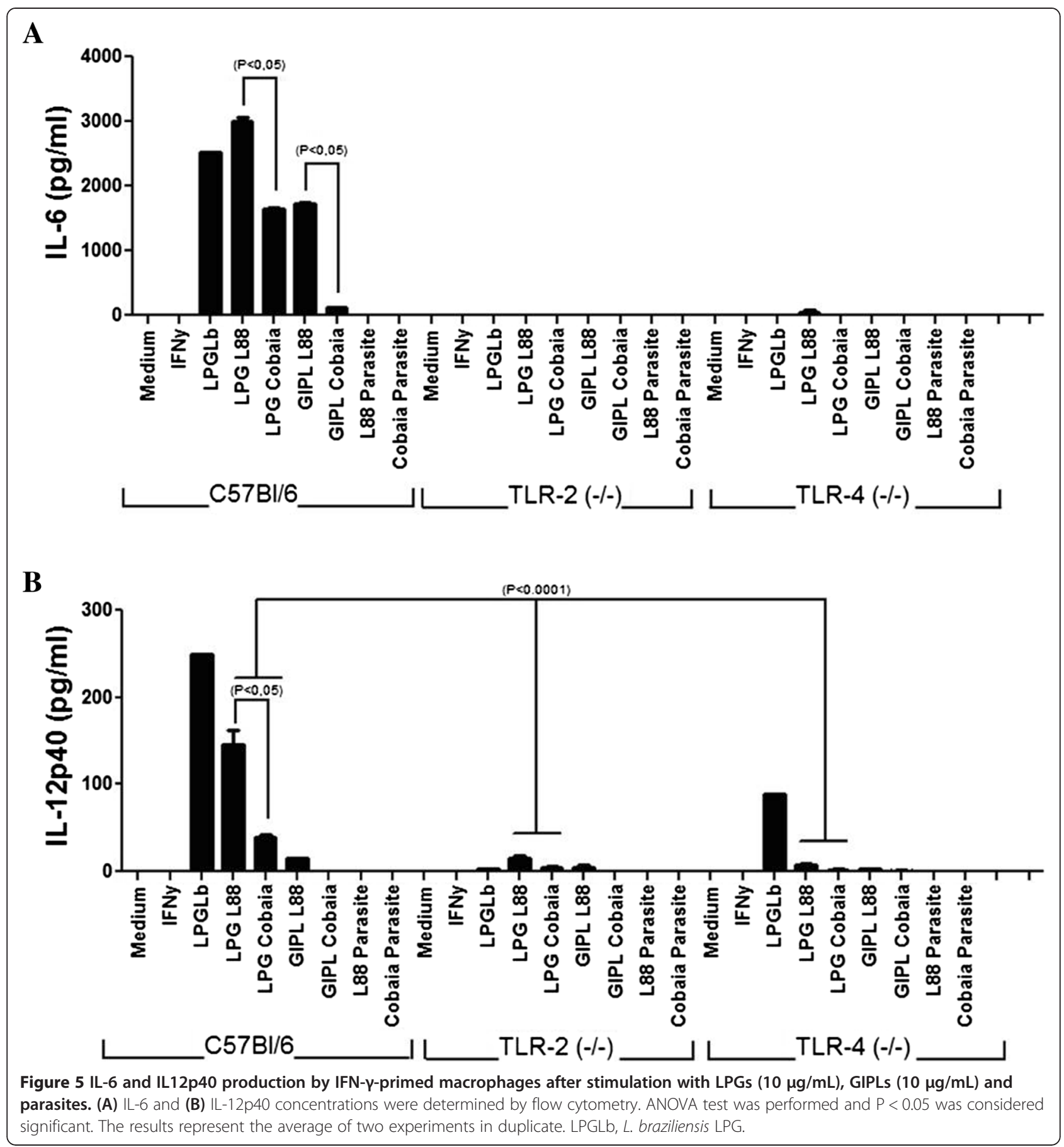

production, receptors involved, signaling pathways and in vivo infectivity for their natural host. This study aimed to enhance our understanding of molecular aspects of L. enriettii biology and glycobiology.

The two L. enriettii strains showed a lower growth pattern in M199 medium in comparison to L. infantum and L. braziliensis from previous studies under the same conditions, never reaching a density above to $1 \times$ $10^{7}$ cells/mL $[33,43]$. Higher densities were observed for the Cobaia strain, although both achieved stationary phase at the tenth day of culture. This indicates that the strains possess different division profiles.

Studies focusing on Leishmania-host interaction are important for understanding parasite biology. Surface glycoconjugates are key factors during this process. They enable successful infection in the hostile environments present both in the vertebrate and invertebrate hosts [8,44]. The LPG is the most studied Leishmania glycoconjugate not 


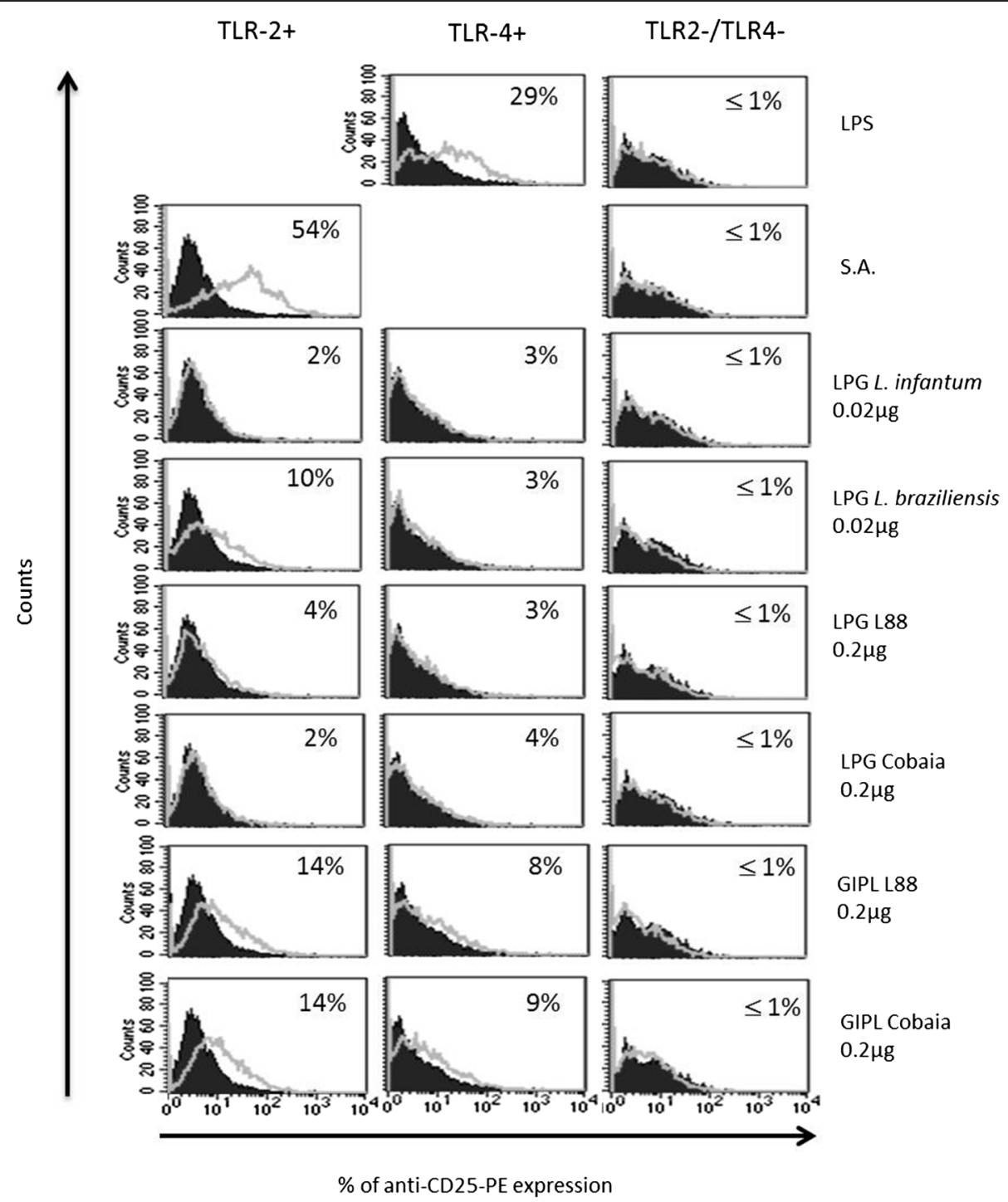

Figure 6 GIPLs purified from two strains of $L$. enriettii (L88 and Cobaia) induce translocation of NF-kB through TLRs. CHO cells expressing TLR2 (TLR2+), TLR4 (TLR4+), or neither (TLR2-/TLR4-) were either untreated (black line) or exposed (gray line) to LPS, S. aureus (SA), L. infantum LPG, L. braziliensis LPG, L. enriettii strain L88 LPG (LPG L88), L. enrietti strain Cobaia LPG (LPG Cobaia), L. enriettii strain L88 GIPL (GIPL L88) or L. enriettii strain Cobaia GIPL (GIPL Cobaia), as indicated. CD25 expression was measured by flow cytometry $18 \mathrm{~h}$ after stimulation. Percentage $=$ percentage of CD25 expression on stimulated cells minus percentage of CD25 expression on non-stimulated cells.

only in the Old World [9,35,45-47], but also in the New World $[8,21,33,42,43,48]$. The LPGs of L. mexicana [48] and $L$. infantum (PP75 strain) have one $\beta$-glucose linked to the repeat units. In L. infantum, this $\beta$-glucose is downregulated in expression after metacyclogenesis [33]. The opposite happens with L. braziliensis, where the procyclic forms do not have side chains and the metacyclic forms have 1-2 $\beta$-glucoses [43]. In this study, the LPG and preliminary GIPL structures of $L$. enriettii were determined. Based on our data, the LPGs were devoid of side chains, being represented by the disaccharide Gal-Man common to all LPGs. The LPGs from the two L. enriettii strains were similar to the LPGs of L. braziliensis (strain M2903),
L. infantum type I and L. donovani (Sudan strain) $[42,43,46]$. A distinguished feature of $L$. enriettii strain L88 was observed in the Western-Blot. Its higher molecular weight is consistent with a longer disaccharide chain as detected by the antibody CA7AE, specific for the Gal-Man repeat units of the LPG [41]. Previous studies with $L$. major and $L$. infantum have shown that more complex LPG structures were able to trigger a higher NO production by macrophages [20,42]. Consistent, with those data, the longer LPG from L. enriettii (L88 strain) exhibited a greater ability to induce $\mathrm{NO}$ production by macrophages. The preliminary structures of $L$. enriettii LPGs are depicted in Figure 10. 

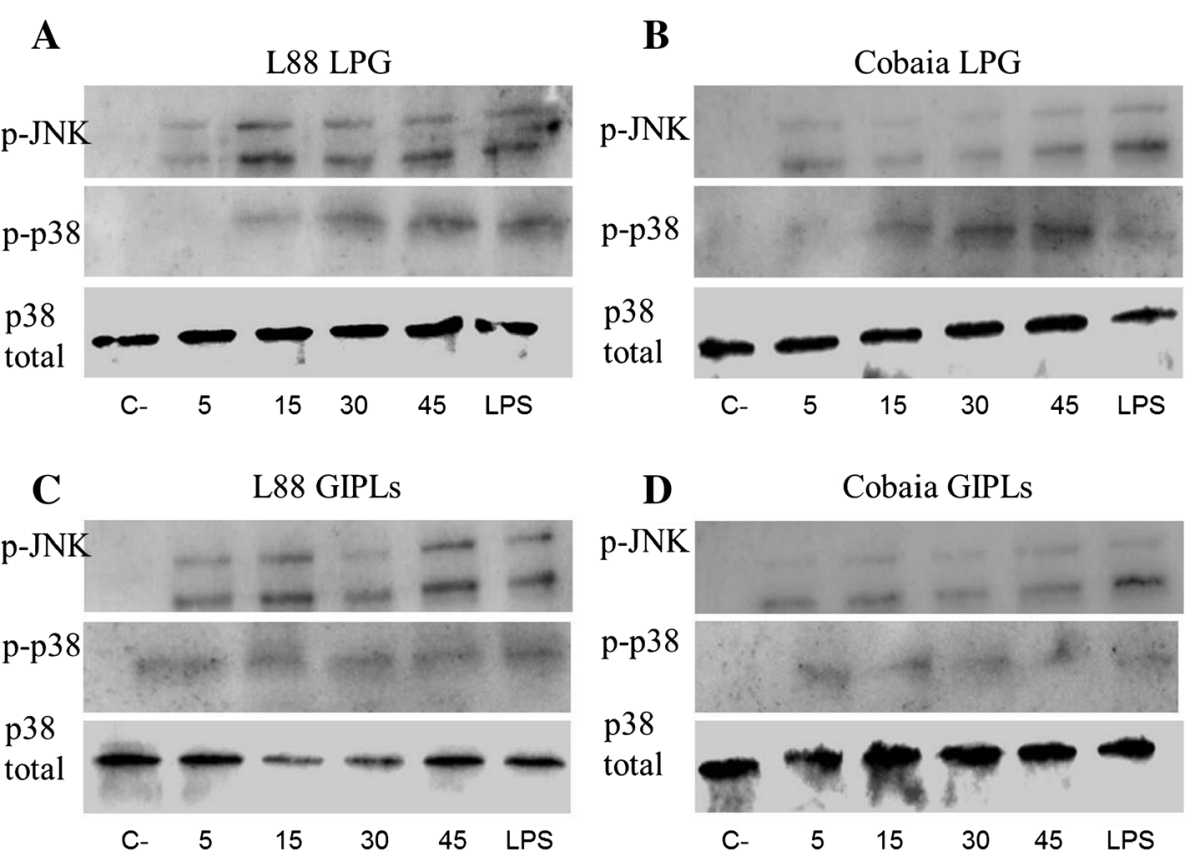

Figure 7 Activation of MAPKs ( $\mathrm{p} 38$ and JNK) by L. enriettii glycoconjugates (LPG and GIPLs) from both strains (L88 and Cobaia) in J774A.1 peritoneal macrophages. Macrophages were stimulated for 5, 15, 30 and 45 min with $10 \mu \mathrm{g} / \mathrm{mL}$ of LPG and GIPLs from L. enriettii L88 $(\mathbf{A}, \mathbf{C})$ and L. enriettii Cobaia (B and D). Dually phosphorylated MAPKs (JNK and p38) were detected by western blot. C-, negative control; Total p38 content was used as the normalizing protein.

Leishmania GIPLs may be classified as type I, II and hybrids [revised by 8]. Type I GIPLs (mannose rich) are found in L. infantum [11], L. donovani, L. tropica and L. aethiopica [45]. The galactose-rich type II GIPLs are commonly found in L. braziliensis [11], L. major [10], L. mexicana [9] and L. panamensis [47]. Finally, hybrid GIPLs have mixed structural features of type I and II. It is found in L. mexicana and L. donovani [9]. Different from LPGs, the GIPLs from the two strains exhibited polymorphisms in their sugar compositions. The L88 strain was galactose rich, suggesting its similarity to a type II GIPL. On the other hand,
Cobaia strain had a composition rich in glucose, a feature never previously observed in any GIPL of Leishmania [11].

The polymorphisms observed and the LPG size and in the GIPL composition from both strains were evaluated in vitro using murine peritoneal macrophages in order to establish their pro-inflammatory properties in the innate immune compartment. Some of those mechanisms were observed in this work during interaction with $L$. enriettii.

In Leishmania, TLR2, TLR4 and TLR9 are the main receptors activated by parasite PAMPs $[8,32,49,50]$.

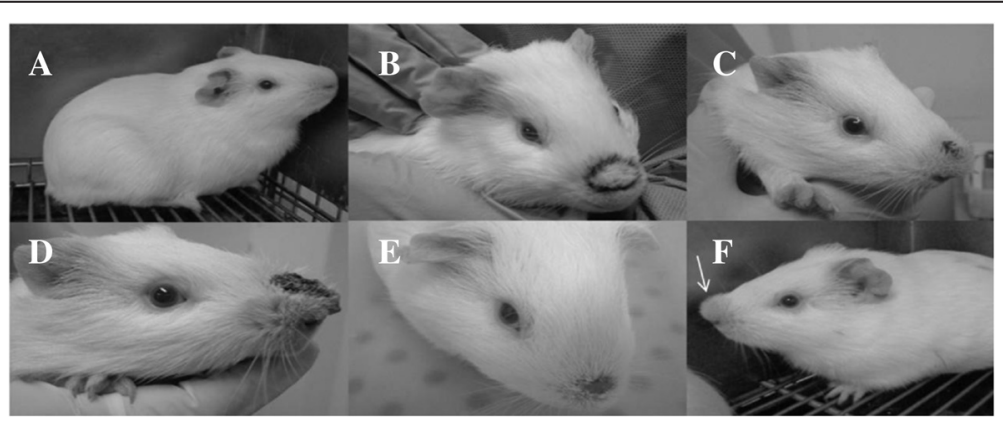

Figure 8 Lesions in C. porcellus infected by L. enriettii strains in the presence/absence of salivary gland extract (SGE) from Lutzomyia longipalpis. Male C. porcellus were inoculated with $1 \times 10^{5}$ parasites of L. enriettii strains (L88 and Cobaia). (A) non-infected C. porcellus; (B) C. porcellus infected with L88 strain (4 weeks of infection); (C) C. porcellus infected with L88 strain (5 weeks infection); (D) C. porcellus infected with L88 strain + SGE (7 weeks of infection); (E) C. porcellus infected with L88 strain (8 weeks of infection) and (F) C. porcellus infected with Cobaia strain (4 weeks of infection). 


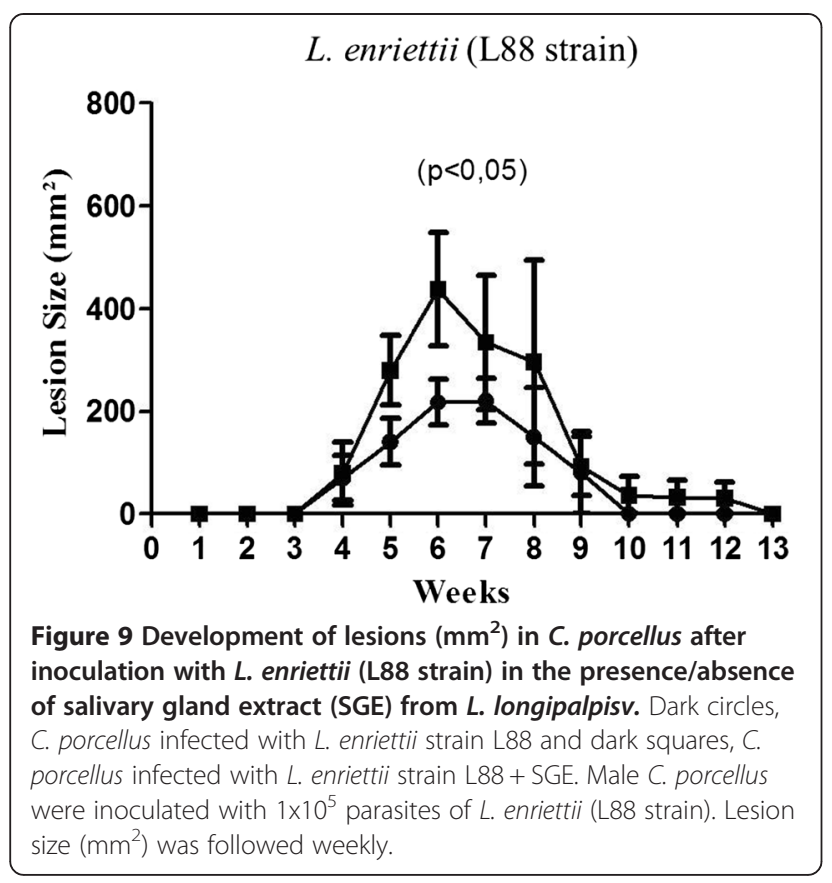

Their activation is dependent on MyD88 adapter protein resulting in the production of nitric oxide and proinflammatory cytokines $[21,23,24]$. Previous studies have shown that Leishmania LPGs and GIPLs are agonists of TLR2 and TLR4, respectively [11,21,23,24]. In L. enriettii glycoconjugates, a differential stimulation pattern was observed by those molecules in murine macrophages, suggesting the participation of both TLR2 and TLR4 by murine macrophages for both strains. Interestingly, a more pro-inflammatory activity was observed for L88 strain in comparison to Cobaia strain not only for LPG, but also for GIPLs. Previous studies using $L$. major, $L$. braziliensis and L. infantum GIPLs have shown the antiinflammatory function of this glycoconjugate thus inhibiting the NO production when stimulated with IFN- $\gamma$ and LPS [11,51]. This profile was not observed in this study, where the two L. enriettii strains were able to trigger the production of $\mathrm{NO}$ and other cytokines. In conclusion, the higher pro-inflammatory activity of the L88 LPG may be related to its size, whereas the higher proinflammatory activity of their GIPLs may be due to the presence of a greater galactose content. Consistent with those data, a similar profile was observed interspecifically with $L$. braziliensis and L. infantum. A higher proinflammatory activity was observed for L. braziliensis LPG and this was due to their ability to translocate NF$\mathrm{kB}$ [21]. Consistent with those observations, JNK activation was more pronounced in L88 LPG than Cobaia LPG. However, we could not detect any substantial difference for p38 after stimulation with LPG and GIPLs from both strains. Confirming this pattern, the ability to activate p38 was also observed for $L$. donovani LPG in J774A.1 macrophages [52]. However, L. braziliensis and $L$. infantum GIPLs were not able to activate murine macrophages [11], a strong indication that this modulation may be species specific.

In the present study, the activation of $\mathrm{NO}$ and cytokines by the LPGs and GIPLs of the two L. enriettii strains was done preferably via TLR2 and secondarily via TLR4. The GIPLs data differ from those observed in $L$. major [51] and L. braziliensis/L. infantum [11], whose activation was mainly through TLR4. Those features are

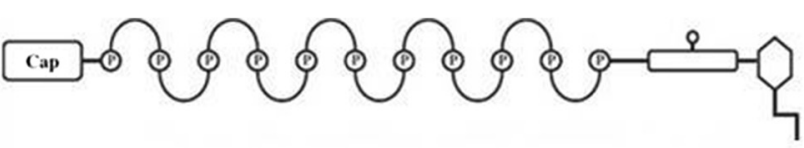

A Procyclic LPG of L. braziliensis M2903 strain (Soares et al., 2005)
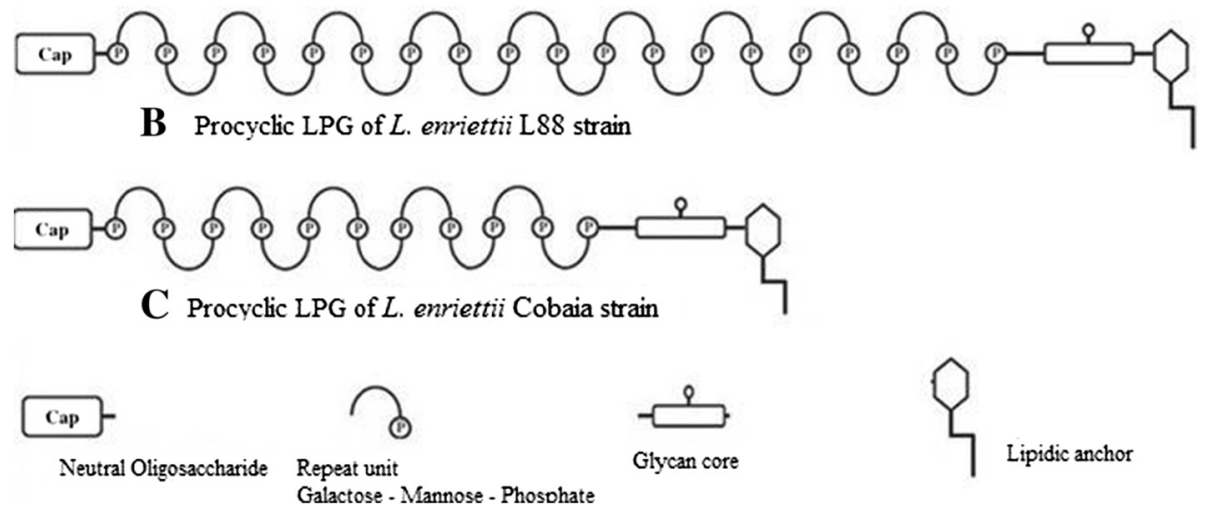

Figure 10 Schematic diagram of $L$. enriettii LPGs. The central portion of the structure is $\mathrm{Gal}(\alpha 1,6) \mathrm{Gal}(\alpha 1,3) \mathrm{Galf}(\alpha 1,3)\left[\mathrm{Gl}\left(\mathrm{C}\left(\mathrm{1}-\mathrm{PO} \mathrm{H}_{4}\right)-6\right] \mathrm{Man}(\mathrm{a} 1,3)\right.$ Man (a1,4)GlcN(a1:6), attached lipid anchor to alkyl-2-lyso-1-O phosphatydylinositol (PI). The repeat units are 6-Gal( $(\beta 1,4)$ Man( $(a 1)-P_{4}$. The precise numbers of the repeat units in the L. enriettii LPGs and the CAP constitution are not known. $P=$ phosphate. 
consistent with the diversity observed in Leishmania species, whose variations in their glycoconjugates may account at least in part for those differences. Since a dual role of TLR2 and TLR4 was observed after stimulation with both glycoconjugates, the next step was to observe their individual role using $\mathrm{CHO}$ cells.

The individual evaluation of the function of each TLR utilizing $\mathrm{CHO}$ cells, showed that the LPGs were not able to activate TLR2 and TLR4 and this feature is very similar to that observed for L. infantum LPG [11]. This is the first time that GIPLs were exposed to $\mathrm{CHO}$ cells. Consistent with our previous results in C57BL/6 mice, the GIPLs were able to activate either TLR2 or TLR4. The fact, that LPGs were not able to separately activate $\mathrm{CHO}$ transfected cells may suggest a co-participation of the two receptors by $L$. enriettii LPGs. It is well known that for lipopeptides of bacteria and GPI-mucins of $T$. cruzi [53], a dimerization between TLR1/6 and TLR2/6 is reported [54]. However, the phenomenon of dimerization between TLR2/4 should be further explored in Leishmania.

Based on the distinguished profile observed in the proinflammatory studies, we examined the infectivity of both strains in its natural host C. porcellus. Previous studies have shown that small amounts of sand fly saliva could promote the infection when inoculated with promastigotes of Leishmania spp [40,55-57]. Consistent with those data, in the animals infected with L88 strain plus SGE, the lesion size was $38.35 \%$ larger and took longer to heal. On the other hand, those infected with the Cobaia strain did not develop ulcerated lesion. Those data confirmed the potential of the L. longipalpis saliva in exacerbate Leishmania infection and the more pro-inflammatory activity of L88 strain which was the only one able to develop lesions in C. porcellus [58-62].

\section{Conclusions}

In conclusion, our results showed that the strains of $L$. enriettii isolated in two distinct periods (1945 and 1985) differ biologically in the parameters studied. The in vitro pro-inflammatory profile and their infectivity in vivo were confirmed in its vertebrate host. A distinguished feature of $L$. enriettii glycoconjugates is that their LPGs could induce a higher production of IL-12 and their GIPLs were very pro-inflammatory. This is very different from what was reported in human pathogenic species such as L. braziliensis and L. infantum $[8,11,21]$. This may lead to the speculation that in the human, the excessive activation of the innate immune system by L. enriettii glycoconjugates may prevent this host to get infected with this parasite. This study is part of a wider project on the glycobiology of New World species of Leishmania.

\section{Competing interests}

The authors declare that they have no competing interests.

\section{Authors' contributions}

LFP, RRA, PMN and RPS performed most of experiments, data analysis, and drafted the manuscript. PMP contributed with in vivo experiments. NLP and MAC performed NF-kB experiments. OAM and ACOS contributed with CBA analysis. JHC and ACT performed MAPKs experiments. NFG and MNM contributed with sand fly experiments and Leishmania enriettii strains maintenance. All authors read and approved the final manuscript.

\section{Acknowledgements}

R.P.P. Soares, M.A. Campos, M.N. Melo and O.A Martins-Filho are supported by Conselho Nacional de Pesquisa e Desenvolvimento. R.P Soares and M.A. Campos are supported by Oswald Cruz Foundation (CNPq/FIOCRUZ) (PAPES $\mathrm{VI}$ ) and Programa Pesquisador Mineiro -Fundação de Amparo a Pesquisa do Estado de Minas Gerais (FAPEMIG), PROEP (401975/2012-6) and INCTV CNPq R.R. Assis (162209/2012-6) and N.L Pessoa are supported by CNPq. This project was approved by the Ethical Committee of Animal Handling (CEUA) from FIOCRUZ, Brazil (Protocol P-9/13-3).

\section{Author details}

${ }^{1}$ Centro de Pesquisas René Rachou, Fundação Oswaldo Cruz, FIOCRUZ, Belo Horizonte, Minas Gerais, Brazil. ²Laboratório de Fisiologia de Insetos Hematófagos, Departamento de Parasitologia, Instituto de Ciências Biológicas, Universidade Federal de Minas Gerais, Belo Horizonte, MG, Brazil. ${ }^{3}$ Laboratório de Biologia de Leishmania, Departamento de Parasitologia, Instituto de Ciências Biológicas, Universidade Federal de Minas Gerais, Belo Horizonte, Brazil. " Laboratório de Imunologia Celular e Bioquímica de Fungos e Protozoários, Departamento de Ciências Biológicas, Campus Diadema, Universidade Federal de São Paulo, UNIFESP, São Paulo, SP, Brazil.

${ }^{5}$ Laboratory of Cellular and Molecular Parasitology, Centro de Pesquisas René Rachou, Fundação Oswaldo Cruz, FIOCRUZ, Av. Augusto de Lima 1715, Belo Horizonte, Minas Gerais 30190-002, Brazil.

Received: 23 August 2014 Accepted: 1 January 2015

Published online: 17 January 2015

\section{References}

1. Machado MI, Milder RV, Pacheco RS, Silva M, Braga RR, Lainson R. Naturally acquired infections with Leishmania enrietti Muniz and Medina 1948 in guinea-pigs from São Paulo, Brazil. Parasitology. 1994;109:135-8.

2. Muniz J, Medina HS. Leishmaniose tegumentar do cobaio (Leishmania enriettii n.sp). Hospital. 1948;33:7-25.

3. Luz E, Giovannoni M, Borba AM. Infecção de Lutzomyia monticola por Leishmania enriettii. An Fac Med Univ Fed Paraná. 1967;9:121-8.

4. Lobato Paraense $V$. The spread of Leishmania enriettii through the body of the guinea pig. Trop Med Hyg. 1953;47:556-66

5. Bryceson AD. Immunity in cutaneous leishmaniasis of the guinea-pig. Clin Exp Immunol. 1970;7:301-41.

6. Schottelius J. Selective lectin reactions of two stocks of Leishmania enriettii with differing pathogenicity. J Parasitol Res. 1987;73:1-8.

7. Dougall AM, Alexander B, Holt DC, Harris T, Sultan AH, Bates PA, et al. Evidence incriminating midges (Diptera: Ceratopogonidae) as potential vectors of Leishmania in Australia. Int J Parasitol. 2011;41:571-9.

8. De Assis RR, Ibraim IC, Nogueira PM, Soares RP, Turco SJ. Glycoconjugates in New World species of Leishmania: polymorphisms in lipophosphogycan and glycoinositolphospholipids and interaction with hosts. Biochim Biophys Acta: General Subjects. 2012:13:1-12.

9. McConville MJ, Ferguson MA. The structure, biosynthesis and function of glycosylated phosphatidylinositols in the parasitic protozoa and higher eukaryotes. J Biochem. 1993;294:305-24.

10. McConville MJ, Homans SW, Thomas-Oates JE, Dell A, Bacic A. Structures of the Glycoinositolphospholipids from Leishmania major. A family of novel galactofuranose-containing glycolipids. J Biol Chem. 1990;265:7385-94.

11. Assis RR, Ibraim IC, Noronha FS, Turco SJ, Soares RP. Glycoinositolphospholipids from Leishmania braziliensis and L. infantum: Modulation of Innate Immune System and Variations in Carbohydrate Structure. PLos Negl Trop Dis. 2012;6:e 1543.

12. Orlandi Jr PA, Turco SJ. Structure of the lipid moiety of the Leishmania donovani lipophosphoglycan. J Biol Chem. 1987;262:10384-91.

13. Bogdan C, Röllinghoff M. How do protozoan parasites survive inside macrophages. Parasitol Today. 1999;15:22-8. 
14. Wilhelm P, Ritter U, Labbow S, Donhauser N, Röllinghoff M, Bogdan C, et al. Rapidly fatal Leishmaniasis in resistant C57BL/6 mice lacking TNF. J Immunol. 2001;166:4012-9.

15. Dermine JF, Scianimanico S, Privé C, Descoteaux A, Desjardins M. Leishmania promastigotes Require Lipophosphoglycan to Actively Modulate the Fusion Properties of Phagosomes at an Early Step of Phagocytosis. Cell Microbiol. 2000;2:115-26.

16. Piedrafita D, Proudfoot L, Nikolaev AV, Xu D, Sands W, Feng GJ, et al. Regulation of macrophage IL-12 synthesis by Leishmania phosphoglycans. Eur J Immunol. 1999;29:235-344.

17. De Carvalho-Vivarini A, Pereira RM, Teixeira KL, Calegari-Silva TC, Bellio M, Laurenti MD, et al. Human Cutaneous Leishmaniasis: Interferon- dependent expression of double-stranded RNA-dependent Protein Kinase (PKR) via TLR2. Faseb J. 2011;25:4162-73.

18. Dermine JF, Goyette G, Houde M, Turco SJ, Desjardins M. Leishmania donovani Lipophosphoglycan disrupts phagosome microdomains in $\mathrm{J774}$ macrophages. Cell Microbiol. 2005;7:1263-70.

19. Brittingham A, Mosser DM. Exploitation of the complement system by Leishmania promastigotas. Parasitol Today. 1996;12:444-7.

20. Proudfoot L, Nikolaev AV, Feng GJ, Wei WQ, Ferguson MA, Brimacombe JS, et al. Regulation of the expression of nitric oxide synthase and leishmanicidal activity by glycoconjugates of Leishmania lipophosphoglycan in murine macrophages. Proc Nat Acad Sci USA. 1996;93:10984-9.

21. Ibraim IC, de Assis RR, Pessoa NL, Campos MA, Melo MN, Turco SJ, et al. Two biochemically distinct lipophosphoglycans from Leishmania braziliensis and Leishmania infantum trigger different innate immune responses in murine macrophages. Parasit Vectors. 2013;6:1-11.

22. Feng GJ, Goodridge HS, Harnett MM, Wei XQ, Nikolaev AV, Higson AP, et al. Extracellular Signal-related Kinase (ERK) and P38 Mitogen-activated Protein (MAP) kinases differentially regulate the Lipopolysaccharide-mediated induction of inducible nitric oxide synthase and IL-12 in macrophages: Leishmania phosphoglycans subvert macrophage $11-12$ production by targeting ERK MAP kinase. J Immunol. 1999;163:6403-12.

23. De Veer MJ, Curtis JM, Baldwin TM, Di Donato JA, Sexton A, McConville MJ, et al. MyD88 is essential for clearance of Leishmania major: possible role for lipophosphoglycan and Toll-like receptor 2 signaling. Eur J Immunol. 2003;33:2822-31.

24. Becker I, Salaiza N, Aguirre M, Delgado J, Carrillo-Carrasco N, Kobeh LG, et al. Leishmania Lipophosphoglycan (LPG) Activates NK Cells Through Toll-like Receptor-2. Mol Biochem Parasitol. 2003;130:65-74.

25. Rojas-Bernabé A, Garcia-Hernández O, Maldonado-Bernal C, DelegadoDominguez J, Ortega E, Gutiérrez-Kobeh L, et al. Leishmania mexicana lipophosphoglycan activates ERK and p38 MAP kinase and induces production of proinflammatory cytokines in human macrophages through TLR2 and TLR4. Parasitology. 2014;141:788-800.

26. Guimarães-Costa AB, Nascimento MT, Froment GS, Soares RP, Morgado FN, Conceição-Silva F, et al. Leishmania amazonensis promastigotes induce and are killed by neutrophil extracellular traps. Proc Natl Acad Sci USA. 2009;106:6748-53.

27. Luz NF, Andrade BB, Feijó DF, Araújo-Santos T, Carvalho GQ, Andrade D, et al. Heme oxygenase-1 promotes the persistence of Leishmania chagasi infection. J Immunol. 2012;188:4460-7.

28. Pimenta PFP, Turco SJ, McConville MJ, Lawyer PG, Perkins P, Sacks DL. Stage-specific adhesion of Leishmania promastigotes to the sandfly midgut. Science. 1992;234:212-4.

29. Pimenta PFP, Saraiva EM, Rowton E, Modi GB, Garraway LA, Beverley SM, et al. Evidence that vectorial competence of phlebotomine sandflies for different species is controlled by structural polymorphisms in the surface of lipophosphoglycan. Proc Nat Acad Sci USA. 1994;91:9155-9.

30. Turco SJ, Sacks DL. Control of Leishmania-sand fly interactions by polymorphisms in lipophosphoglycan structure. Meth Enzymol. 2003;363:377-81.

31. Kamhawi S, Ortigao MR, Pham VM, Kumar S, Lawyer PG, Turco SJ, et al. A role for insect galectins in parasite survival. Cell. 2004;119:329-41.

32. Tuon FF, Amato VS, Bacha HA, Almusawi T, Duarte MI, Amato Neto V. Toll like receptors and leishmaniasis. Infect Immun. 2008;76:866-72.

33. Soares RP, Macedo ME, Ropert C, Gontijo NF, Almeida IC, Gazzinelli RT, et al. Leishmania chagasi: lipophosphoglycan characterization and binding to the midgut of the sandfly vector Lutzomyia longipalpis. Mol Biochem Parasitol. 2002;121:213-24
34. Dubois M, Gilles K, Hamilton JK, Rebers PA, Smith F. Colorimetric method for determination of sugar and related substances. Anal Chem. 1956;28:350-6.

35. Soares RP, Barron T, McCoy-Simandle K, Svobodova M, Warburg A, Turco SJ. Leishmania tropica: intraspecific polymorphisms in lipophosphoglycan correlate with transmission by different Phlebotomus species. Exp Parasitol. 2004; 107:105-14

36. Kolodziej H, Radtke OA, Kiderlen AF. Stimulus (polyphenol, IFN-gamma, LPS)-dependent nitric oxide production and antileishmanial effects in RAW 264.7 macrophages. Phytochemistry. 2008;69:3103-10.

37. Lien E, Sellati TJ, Yoshimura A, Flo TH, Rawadi G, Finberg RW, et al. Toll-like receptor 2 functions as a pattern recognition receptor for diverse bacterial products. J Biol Chem. 1999;274:33419-25.

38. Delude RL, Yoshimura A, Ingalls RR, Golenbock DT. Construction of a lipopolysaccharide reporter cell line and its use in identifying mutants defective in endotoxin, but not TNF-alpha, signal transduction. J Immunol. 1998;161:3001-9.

39. Modi GB, Tesh RB. A simple technique for mass rearing Lutzomyia longipalpis and Phlebotomus papatasi (Diptera: Psychodidae) in the laboratory. J Med Entomol. 1983;20:568-9.

40. Belkaid Y, Kamhawi S, Modi G, Valenzuela J, Noben-Trauth N, Rowton E, et al. Development of a natural model of cutaneous leishmaniasis: powerful effects of vector saliva and saliva pre exposure on the long-term outcome of Leishmania major infection in the mouse ear dermis. J Biol Chem. 1998;188:1941-53.

41. Tolson DL, Turco SJ, Beecroft RP, Pearson TW. The immunochemical structure and surface arrangement of Leishmania donovani lipophosphoglycan determined using monoclonal antibodies. Mol Biochem Parasitol. 1989;35:109-18

42. Coelho-Finamore JM, Freitas VC, Assis RR, Melo MN, Novozhilova N, Secundino NF, et al. Leishmania infantum: lipophosphoglycan intraspecific variation and interaction with vertebrate and invertebrate hosts. Int J Parasitol. 2011:41:811-20.

43. Soares RP, Cardoso TL, Barron T, Araújo MS, Pimenta PF, Turco SJ. Leishmania braziliensis: a novel mechanism in the lipophosphoglycan regulation during metacyclogenesis. Int J Parasitol. 2005;35:245-53.

44. Sacks D, Kamhawi S. Molecular aspects of parasite-vector and vector-host interactions in Leishmaniasis. Annu Rev Microbiol. 2001;55:453-83.

45. Schneider P, Schnur LF, Jaffe CL, Ferguson MA, McConville MJ. Glycoinositolphospholipid profiles of four serotypically distinct old world Leishmania strains. J Biochem. 1994;304:603-9.

46. Sacks DL, Pimenta PF, McConville MJ, Schneider P, Turco SJ. Stage-specific binding of Leishmania donovani to the sand fly vector midgut is regulated by conformational changes in the abundant surface lipophosphoglycan. J Exp Med. 1995;181:685-97.

47. Zawadzki J, Scholz C, Currie G, Coombs GH, McConville MJ. The glycoinositolphospholipids from Leishmania panamensis contain unusual glycan and lipid moieties. J Mol Biol. 1998;2:287-99.

48. Ilg T, Etges R, Overath P, McConville MJ, Thomas-Oates J, Thomas J, et al Structure of Leishmania mexicana lipophosphoglycan. J Biol Chem. 1992;267:6834-40.

49. Vargas-Inchaustegui DA, Tai W, Xin L, Hogg AE, Corry DB, Soong L. Distinct roles for MyD88 and Toll-like receptor 2 during Leishmania braziliensis infection in mice. Infect Immun. 2009;77:2948-56.

50. Faria MS, Reis FC, Lima AP. Toll- like receptors in Leishmania infections: guardians or promoters? J Parasitol Res. 2012;10:1-12.

51. Proudfoot L, O'Donnell CA, Liew FY. Glycoinositolphospholips of Leishmania major inhibit nitric oxide synthesis and reduce leishmanicidal activity in murine macrophages. Eur J Immunol. 1995;25:745-50.

52. Balaraman S, Singh VK, Tewary P, Madhubala R. Leishmania lipophosphoglycan activates the transcription factor activating protein 1 in J774A.1 macrophages through the extracellular signal-related kinase (ERK) and p38 mitogen-activated protein kinase. Mol Biochem Parasitol. 2005;139:117-27.

53. Gazzinelli RT, Denkers EY. Protozoan encounters with Toll-like receptor signalling pathways: implications for host parasitism. Nat Rev Immunol. 2006;6:895-906.

54. Kumagai Y, Akira S. Identification and functions of pattern-recognition receptors. J Allergy Clin Immunol. 2010;125:985-92.

55. Titus RG, Ribeiro JM. Salivary gland lysates from the sand fly Lutzomia longipalpis enhance Leishmania infectivity. Science. 1988;239:1306-8. 
56. Laurenti MD, da Matta VL, Pernichelli T, Secundino NF, Pinto LC, Corbett CE, et al. Effects of salivary gland homogenate from wild-caught and laboratory-reared Lutzomyia longipalpis on the evolution and immunomodulation of Leishmania (Leishmania) amazonensis infection. Scand J Immunol. 2009;70:389-95.

57. Laurenti MD, Silveira VM, Secundino NF, Corbett CE, Pimenta PP. Saliva of laboratory-reared Lutzomyia longipalpis exacerbates Leishmania (Leishmania) amazonensis infection more potently than saliva of wild-caught Lutzomyia longipalpis. Parasitol International. 2009;58:220-6.

58. Brown SJ, Rosalsky JH. Blood leukocyte response in hosts parasitized by the hematophagous arthropods Triatoma protracta and Lutzomyia longipalpis. Am J Trop Med Hyg. 1984;33:499-505.

59. Theodos CM, Ribeiro JM, Titus RG. Analysis of enhancing effect of sand fly saliva on Leishmania infection in mice. Infect Immun. 1991;59:1592-8.

60. Sádlová J. The life history of Leishmania (Kinetoplastida: Trypanosomatidae). Acta Soc Zool Bohem. 1999;63:331-66.

61. Donnelly KB, Lima HC, Titus RG. Histologic characterization of experimental cutaneous leishmaniasis in mice infected with Leishmania braziliensis in the presence or absence of sand fly vector salivary gland lysate. J Parasitol. 1998:84:97-103.

62. Melo MN, Williams P, Tafuri WL. Influence of lysates of the salivary glands of Lutzomyia longipalpis on the development of a Leishmania major-like parasite in the skin of the golden hamster. Ann Trop Med Parasitol. 2001;95:59-68.

\section{Submit your next manuscript to BioMed Central and take full advantage of:}

- Convenient online submission

- Thorough peer review

- No space constraints or color figure charges

- Immediate publication on acceptance

- Inclusion in PubMed, CAS, Scopus and Google Scholar

- Research which is freely available for redistribution 